\title{
The Virulence Function and Regulation of the Metalloprotease Gene prtA in the Plant-Pathogenic Bacterium Burkholderia glumae
}

\author{
Tiago Lelis, Jingyu Peng, Inderjit Barphagha, Ruoxi Chen, and Jong Hyun Ham ${ }^{\dagger}$ \\ Department of Plant Pathology and Crop Physiology, Louisiana State University Agricultural Center, Baton Rouge, LA 70803, \\ U.S.A.
}

Accepted 26 January 2019.

\begin{abstract}
Bacterial panicle blight caused by Burkholderia glumae is a major bacterial disease of rice. Our preliminary RNA-seq study showed that a serine metalloprotease gene, prtA, is regulated in a similar manner to the genes for the biosynthesis and transport of toxoflavin, which is a known major virulence factor of $B$. glumae. prtA null mutants of the virulent strain $B$. glumae 336gr-1 did not show a detectable extracellular protease activity, indicating that prtA is the solely responsible gene for the extracellular protease activity detected from this bacterium. In addition, inoculation of rice panicles with the $p r t A$ mutants resulted in a significant reduction of disease severity compared with the wild-type parent strain, suggesting the requirement of prtA for the full virulence of $B$. glumae. A double mutant deficient in both serine metalloprotease and toxoflavin ( $\Delta$ tox $\left.A / \mathrm{prtA}^{-}\right)$exhibited a further numeric but not statistically significant decrease of disease development compared with the $\Delta$ tox $A$ strain. Both the prtA-driven extracellular protease activity and the toxoflavin production were dependent on both the tofI/tofR quorum-sensing and the global regulatory gene $q s m R$, indicating the important roles of the two global regulatory factors for the bacterial pathogenesis by this pathogen.
\end{abstract}

Keywords: bacterial panicle blight of rice, Burkholderia glumae, metalloprotease, quorum-sensing, $q s m R$, virulence factor

Current address for Jingyu Peng: Department of Plant, Soil, and Microbial Sciences, Michigan State University, East Lansing, MI 48824, U.S.A.

Current address for Ruoxi Chen: VSP Vision Care, 3333 Quality Drive, Rancho Cordova, CA 95670, U.S.A.

${ }^{\dagger}$ Corresponding author: J. H. Ham; jham@agcenter.lsu.edu

Funding: This work was supported by the United States Department of Agriculture National Institute of Food and Agriculture (Hatch Project \#1015305) and the Ministry of Agriculture Food and Rural Affairs, Republic of Korea (Strategic Initiative for Microbiomes in Agriculture and Food \#916009021SB010). T. Lelis was supported by the Conselho Nacional de Desenvolvimento Científico e Tecnológico Science Without Border program of the Brazilian government.

*The $\boldsymbol{e}$-Xtra logo stands for "electronic extra" and indicates that six supplementary figures and three supplementary tables are published online.

The author(s) declare no conflict of interest.

๑) 2019 The American Phytopathological Society
Bacterial panicle blight (BPB) is one of the most severe bacterial diseases of rice (Ham et al. 2011). Since first reported in 1956 in Japan (Goto and Ohata 1956), this disease has been reported in many rice-producing areas worldwide (Cui et al. 2016; Ham et al. 2011). Burkholderia glumae, known as a seedborne pathogen, is the major causal agent of BPB, which can survive both internally and externally of rice seeds (Nandakumar et al. 2009; Tsushima 1996). This pathogen also epiphytically grows on rice plants during the booting stage and multiplies on the surface of emerging panicles (Goto 1992).

Infection of rice plant by $B$. glumae is a multifactorial process requiring numerous virulence factors such as toxoflavin, lipase, type III secretion effectors, and flagella (Ham et al. 2011). Toxoflavin, a phytotoxin, is one of the most important virulence factors of $B$. glumae, and the genes for toxoflavin biosynthesis and transport are encoded by the tox $A B C D E$ and toxFGHI operons, respectively (Kim et al. 2004). Toxoflavindeficient strains exhibit substantially lower levels of virulence compared with the wild-type parent but still retain the ability to cause disease in rice (Kim et al. 2004). Substantial reduction of virulence was also reported with the $B$. glumae strains deficient in lipase production (Devescovi et al. 2007) or flagella formation (Kim et al. 2007). Moreover, B. glumae is able to elicit hypersensitive responses in tobacco leaves in a type III secretion system (T3SS)-dependent manner (Kang et al. 2008). Type III effectors also contribute to the virulence of $B$. glumae, as indicated by the reduced virulence of a T3SS-defective mutant, $\Delta h r p B$ (Kang et al. 2008).

In $B$. glumae, major virulence factors are regulated by the tofI/tofR quorum-sensing (QS) system, which is mediated by the luxI and luxR homologs tofI and tofR, respectively (Chun et al. 2009; Kim et al. 2004, 2007). tofI encodes an acylhomoserine lactone synthase responsible for synthesizing the QS signal compound, $N$-octanoyl homoserine lactone (C8HSL), and tofR encodes a cognate receptor that C8-HSL binds to (Dunlap 1999; Kim et al. 2004). The tofI/tofR QS system regulates the expression of toxR and toxJ, which are additional regulatory elements required for the biosynthesis and transport of toxoflavin (Kim et al. 2004, 2007). The tofI/tofR QS system is also essential for flagellar biogenesis and lipase activity in B. glumae (Devescovi et al. 2007; Kim et al. 2007). Nickzad et al. (2015) reported that the tofI/tofR QS also regulates rhamnolipid production, which is required for swarming motility.

$q s m R$, which encodes an IclR-family transcriptional factor was first identified in B. glumae BGR1 as a QS 'master regulator' that controls flagellar biogenesis and flagellum-mediated motility (Kim et al. 2007). In B. glumae BGR1, qsmR exerts its 
regulatory function through $f l h D / f l h C$ and its expression is dependent on the tofI/tofR QS (Jang et al. 2014; Kim et al. 2007). Disruption of $q s m R$ also caused substantial reduction of toxoflavin in liquid Luria Bertani (LB) medium but no difference was observed under the LB agar medium condition, indicating the regulatory role of $q s m R$ in the toxoflavin production in a growth condition-dependent manner (Kim et al. 2007). Previous studies with B. glumae BGR1 also revealed that $q \sin R$ plays a pivotal role in the QS-dependent regulation of metabolic pathways to survive in stress conditions including the high cell density at the stationary phase (An et al. 2014; Goo et al. 2017). Most importantly, the nearavirulent phenotype of the $q s m R$ null mutant observed in the same study indicates that $q s m R$ is a key regulatory element governing the bacterial pathogenesis by B. glumae (Kim et al. 2007).

Our preliminary RNA-seq study with B. glumae 336gr-1 (a virulent strain in the United States) showed a serine metalloprotease gene being downregulated in a B. glumae mutant derivative deficient in the tofI/tofR QS system (Supplementary Table S1). The serine metalloprotease gene corresponds to 'bglu_1g16590' of the reference strain, BGR1, and was named prtA. In this study, we investigated the role of $p r t A$ in the extracellular protease activity and the virulence of B. glumae as well as its regulation by the tofI/tofR QS system and $q s m R$.

\section{RESULTS}

prtA encoding a serine metalloprotease was solely for the extracellular protease activity of B. glumae 336gr-1.

According to the genome sequence information of $B$. glumae BGR1 (Lim et al. 2009) and B. glumae 336gr-1 (Francis et al. 2013), prtA (bglu_1g16590) is located between the putative genes encoding glycogen synthase (bglu_1g16580) and the $\alpha / \beta$ hydrolase fold protein (bglu_1g16600) (Fig. 1A). B. glumae 336gr-1 exhibited a distinct extracellular protease activity on nutrient agar (NA) supplemented with $1 \%$ skim milk. The bacterial extracellular protease activity is indicated by the clear zone surrounding the bacterial colony as a result of protein digestion (Fig. 1B). To test if prtA contributes to the extracellular protease activity of $B$. glumae $336 \mathrm{gr}-1$, null mutants of prtA were generated through insertional mutagenesis, using an

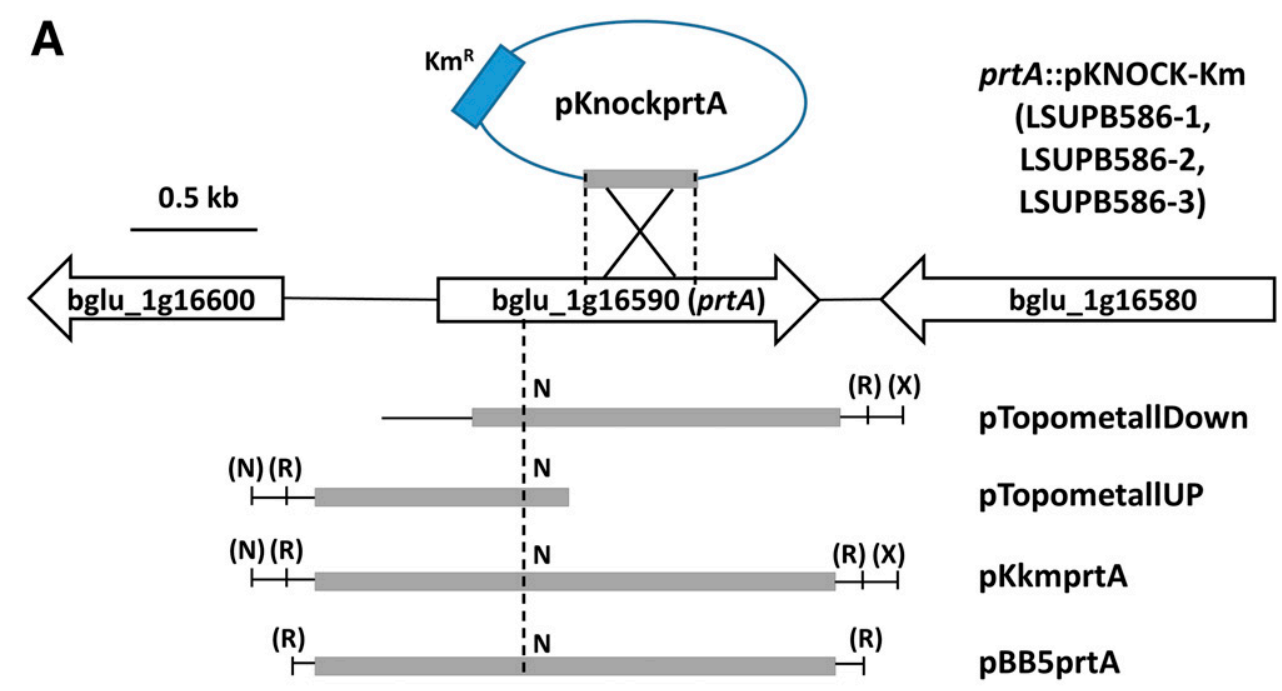

B

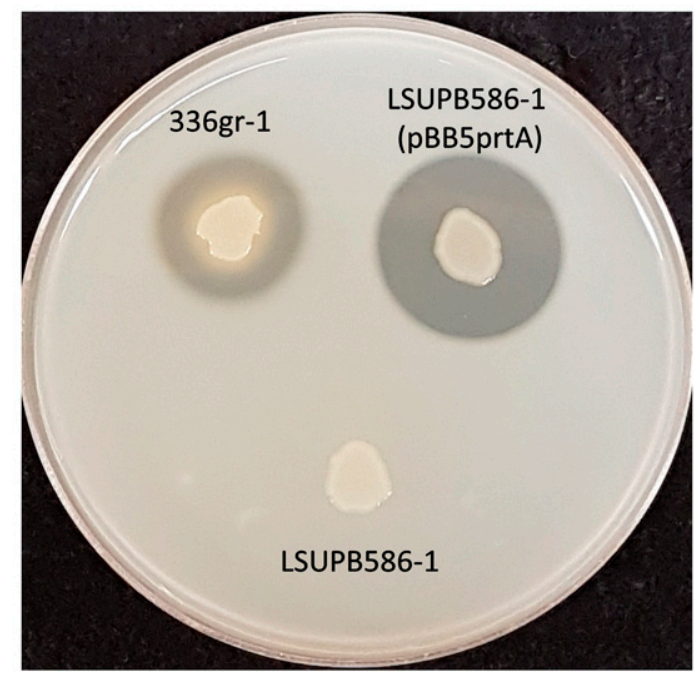

Fig. 1. The role of the prtA gene in the extracellular protease activity of Burkholderia glumae 336gr-1. A, A physical map of prtA that depicts its location, clones, and null mutants. The arrows represent the direction of the size of the corresponding genes, which are indicated by the gene ID numbers of the reference genome B. glumae BGR1 (Lim et al. 2009). B, Extracellular protease activities of 336gr-1 (wild type), a prtA mutant (LSUPB586-1), and LSUPB586-1 carrying pBB5prtA (a prtA clone) on a nutrient agar plate supplemented with 1\% skim milk. Restriction sites used for the DNA constructs are NotI (N), EcoRI $(\mathrm{R})$, and $\mathrm{Xba \textrm {I }}(\mathrm{X})$. Restriction sites of the cloning vectors are presented in parentheses. 
internal fragment of prtA cloned in the suicide vector pKNOCK-Km (Fig. 1A). As shown in Figure 1B, LSUPB5861 , a representative $p r t A^{-}$derivative of $336 \mathrm{gr}-1$, did not exhibit any detectable extracellular protease activity on the NA supplemented with $1 \%$ skim milk. The lost extracellular protease activity in LSUPB586-1 was restored by a prtA clone, pBB5prtA, indicating that prtA is the solely responsible gene for the extracellular protease activity of B. glumae $336 \mathrm{gr}-1$ (Fig. 1B).

The deduced protein sequence of PrtA contained a typical $\mathrm{N}$-terminal signal sequence and its extracellular protease activity was dependent on the type II secretion system (T2SS).

Analysis of the deduced PrtA amino-acid sequence, using SignalP-4.1 (Petersen et al. 2011), revealed an N-terminal secretion signal peptide (Supplementary Fig. S1A). The cleavage site was predicted to be between the 27th (Ala) and the 28th (Gln) amino acid, with maximum $\mathrm{C}$ and $\mathrm{Y}$ scores of 0.801 and 0.869 , respectively, at Q28 and maximum S score of 0.987 at L17. The mean S and D scores of the predicted signal peptide (the first 27 amino acids) were 0.949 and 0.907 , respectively. As substrates of a T2SS need a signal peptide to pass through the inner membrane (Sandkvist, 2001), the PrtA metalloprotease is very likely to be secreted via a T2SS. According to the genome analysis using the web-based bioinformatics tool The Seed Viewer (Overbeek et al. 2005), B. gluame contains at least one gene cluster for a T2SS (Supplementary Fig. S2). To test the requirement of the T2SS for the extracellular activity of PrtA, the $g s p D$ and $g s p E$ genes that encode key components of the T2SS were disrupted individually through single homologous recombination on each gene (Supplementary Fig. S3). Both $g s p D$ and $g s p E$ mutants exhibited substantially less protease activity compared with the wild type, indicating that the extracellular activity of PrtA is dependent on secretion via the T2SS (Fig. 2A and B).

The extracellular protease activity of $B$. glumae 336gr-1 was dependent on the tofI/tofR QS system.

As major virulence factors of B. glumae (i.e., toxoflavin, lipase, flagella) are regulated by the tofI/tofR QS, we further investigated if the extracellular protease activity is under the regulation of tofI/tofR QS. In the genome of B. glumae, tofI and tof $R$ are adjacent to each other with another regulatory gene tof $M$ between the two QS genes (Chen et al. 2012) (Fig. 3A). The four different deletion mutants, LSUPB145 ( $\Delta$ tofI), LSUPB169 ( $\Delta$ tofR), LSUPB286 ( $\Delta$ tofM), and LSUPB139 ( $\Delta$ tofI-tofR) (Chen et al. 2012) (Fig. 3A), were tested for their extracellular protease activities along with the wild-type strain 336gr-1 (Fig. 3B and C). As shown in Figure 3B and C, the extracellular protease activity of $B$. glumae $336 \mathrm{gr}-1$ was not significantly different from the $\Delta$ tofI, $\Delta$ tofR, or $\Delta$ tofM derivative. However, the extracellular protease activity was not detectable in the same conditions with LSUPB139, a derivative of 336gr-1 lacking the entire tofI/tofM/tofR cluster (Fig. 3B and $\mathrm{C})$, and this protease-deficient phenotype of the mutant was restored by pBBtofIMR, a plasmid carrying tofI/tofM/tofR (Supplementary Fig. S4).

\section{prtA was required for the full virulence}

of $B$. glumae 336gr-1.

Virulence function of prtA for B. glumae in rice was examined with three independent $p r t A^{-}$derivatives of 336gr-1, LSUPB586-1, LSUPB586-2, and LSUPB586-3, comparing them with wild-type parent 336gr-1 (Fig. 4A). All the prtA derivatives tested exhibited a significant reduction in disease severity (Fig. 4A). Further tests to verify the virulence function of prtA were conducted with additional strains, including LSUPB586-1(pBB5prtA) (a prtA ${ }^{-}$strain complemented with the prtA clone pBB5prtA), LSUPB537 (a $\Delta$ toxA derivative of $336 \mathrm{gr}-1$ defective in toxoflavin production), and LSUPB633 (a $\Delta$ tox $\mathrm{A} / \mathrm{prtA}^{-}$derivative of $336 \mathrm{gr}-1$ defective in both toxoflavin production and extracellular protease activity). As shown in Figure 4B, the $p r t A^{-}$strain LSUPB586-1 was significantly less virulent than the wild-type parent 336gr-1, while LSUPB5861(pBB5prtA) exhibited a level of virulence comparable to the wild type. This result indicates that the prtA clone pBB5prtA restored the impaired virulence of the prtA mutant, verifying the requirement of the prtA gene for the full virulence of B. glumae 336gr-1. The $\Delta$ toxA strain LSUPB537 was significantly less virulent than the prtA $A^{-}$strain LSUPB586-1, indicating that toxoflavin has a greater impact on the virulence of B. glumae than the PrtA protease (Fig. 4B). Moreover, the double mutant strain LSUPB633 defective in both toxoflavin production and extracellular protease activity exhibited a further reduction in virulence compared with the $\Delta$ toxA strain LSUPB537, although the level of reduction was not statistically significant (Fig. 4B).
A

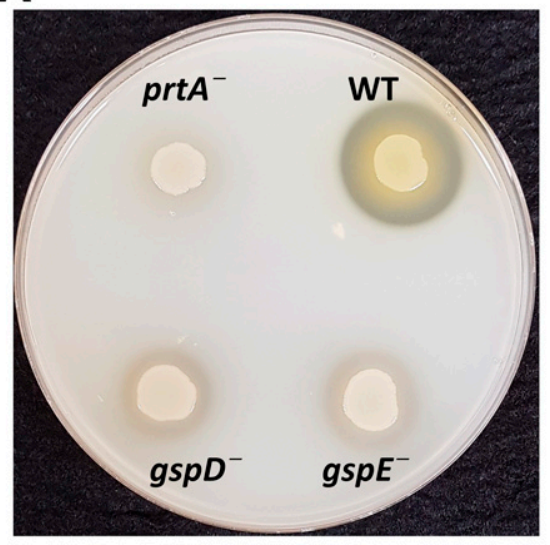

B

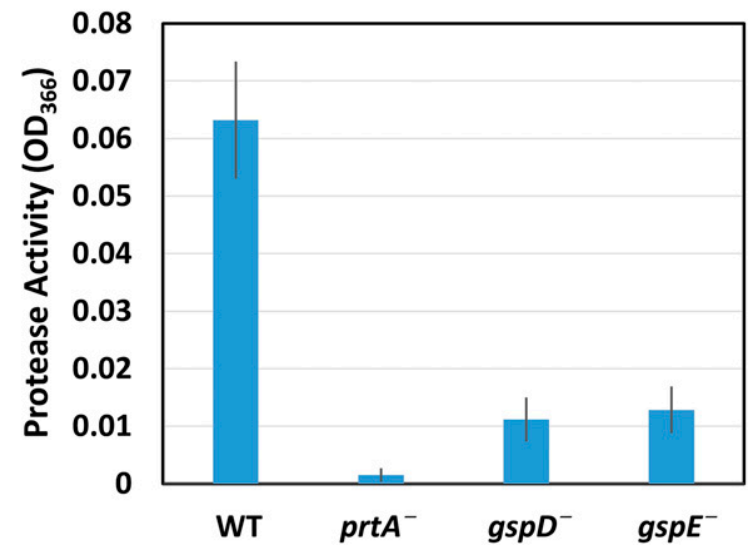

Fig. 2. Extracellular protease activities of Burkholderia glumae 336gr-1 and its gspD and gspE null mutant derivatives. A, Extracellular protease activities of wild type (WT, 336gr-1) and its $\mathrm{gspD}^{-}$(LSUPB640) and $\mathrm{gspE}^{-}$(LSUPB641) derivatives on a nutrient agar plate supplemented with $1 \%$ skim milk. B, Quantified protease activities of the cell-free culture filtrates from the same set of B. glumae strains grown in Luria Bertani broth. Protease activity was quantified following the method of Chessa et al. (2000). LSUPB586-1, a prtA ${ }^{-}$strain, was used as a negative control. 
tox $J$ and toxR, the regulatory genes required for toxoflavin production, were not involved in the extracellular protease activity of PrtA.

In the previous study, by Kim et al. (2004), with B. glumae BGR1, toxJ and toxR were shown to activate the genes for toxoflavin biosynthesis and transport under the regulation of the tofI/tofR QS genes. To determine if toxJ and toxR are also involved in the extracellular protease activity of $B$. glumae $336 \mathrm{gr}-1$, the $\Delta$ toxJ and $\Delta$ tox $R$ derivatives of B. glumae $336 \mathrm{gr}-1$ (named LSUPB592 and LSUPB590, respectively) were generated and their extracellular protease activities were examined. Both $\Delta$ toxJ and $\Delta$ tox $R$ strains exhibited comparable levels of extracellular protease activities to the wild type regardless of solid or liquid medium condition, while the negative control (the QS-deficient mutant LSUPB139) did not express any observable extracellular protease activity (Fig. 5A and B). In contrast, toxoflavin production was abolished in the $\Delta$ toxJ and $\Delta$ toxR strains like in the QS-deficient mutant (Fig. 5C and D), which was congruent with the previous study with $B$. glumae BGR1 (Kim et al. 2004). These results indicate that the extracellular protease activity of B. glumae $336 \mathrm{gr}-1$ is not dependent on the regulatory genes for toxoflavin production, toxJ and toxR, while the tofI/tofR QS genes globally regulate both virulence functions.

\section{$q s m R$ was required for both extracellular}

protease activity and toxoflavin production.

$q s m R$ encoding an IclR-family protein is another regulatory element known to play a crucial role for the virulence of B. glumae BGR1 (Kim et al. 2007). In this study with B. glumae $336 \mathrm{gr}-1$, the $\Delta q s m R$ derivative LSUPB574 completely lost its extracellular protease activity and toxoflavin production, like

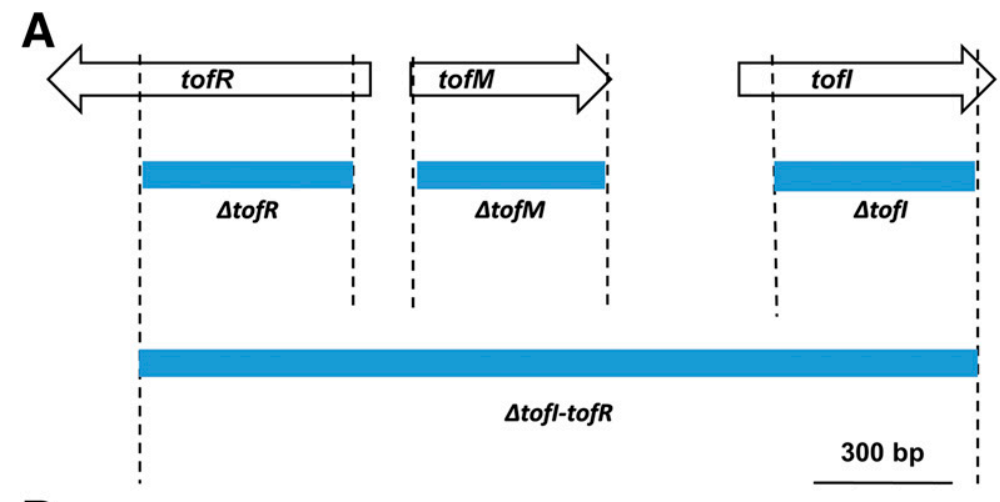

B
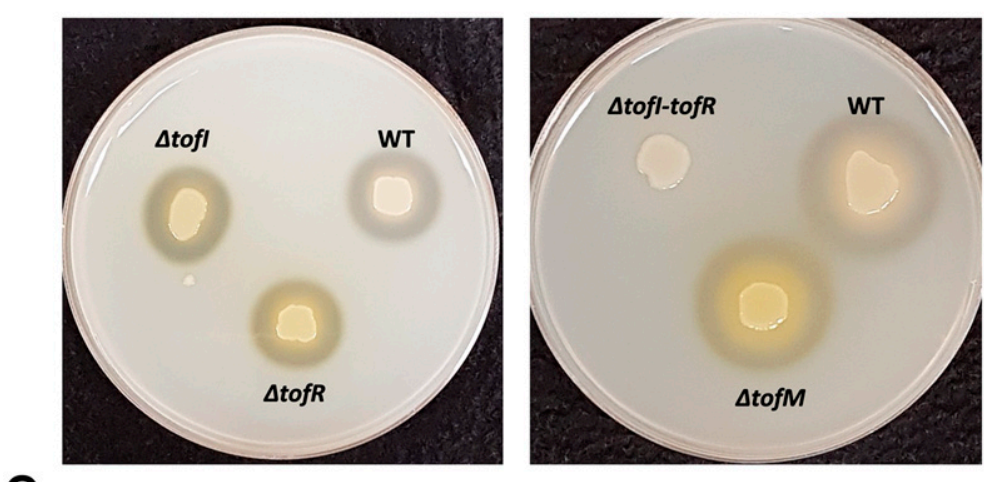

C

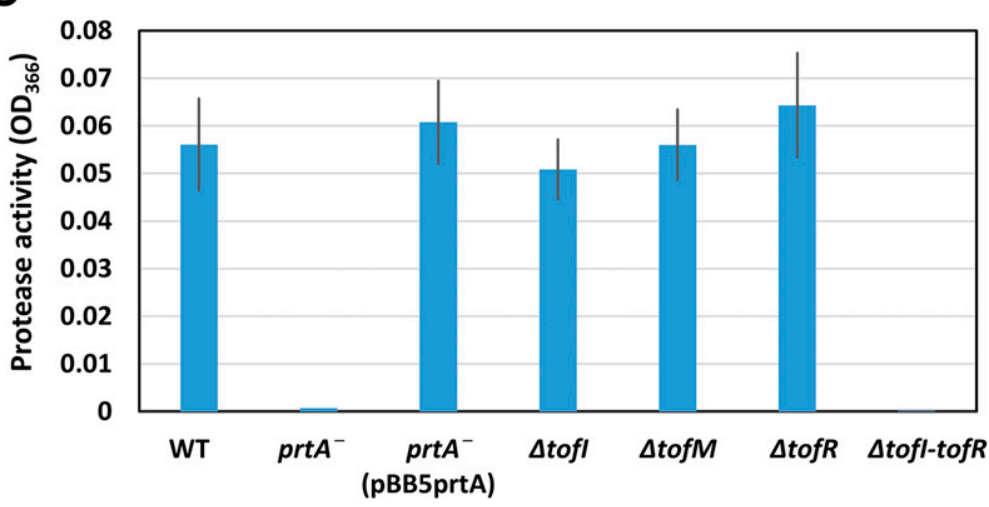

Fig. 3. Extracellular protease activities of Burkholderia glumae 336gr-1 and its quorum-sensing mutant derivatives. A, A schematic description of the quorumsensing mutants tested in this study. B, Extracellular protease activities of the wild type (WT) and the quorum-sensing mutant strains on nutrient agar plates supplemented with $1 \%$ skim milk. C, Quantified protease activities of the cell-free culture filtrates from the same set of B. glumae strains grown in Luria Bertani broth. Protease activity was quantified following the method of Chessa et al. (2000). The strain for each genotype is $336 \mathrm{gr}-1$ (WT), LSUPB145 ( $\Delta$ tofI), LSUPB286 ( $\Delta t o f M)$, LSUPB169 ( $\Delta t o f R)$, and LSUPB139 ( $\Delta t o f I-t o f R)$. LSUPB586-1, a prtA ${ }^{-}$strain, was used as a negative control, and pBB5prtA is a prtA clone that complements the prtA mutant. 
the QS-deficient mutant LSUPB139 (Fig. 6A, B, and C). In contrast, LSUPB145 and LSUPB169 (the $\Delta$ tofI and $\Delta$ tofR derivatives of $B$. glumae $336 \mathrm{gr}-1$, respectively) retained the wildtype level of extracellular protease activity in both NA agar and LB broth medium conditions (Fig. 6A and B), although their toxoflavin production was substantially reduced when they were grown in LB broth (Fig. 6C). Quantitative reverse transcription-polymerase chain reaction (qRT-PCR) tests revealed that the transcription of prtA was abolished in
LSUPB139 ( $\Delta$ tofI-tofR) and LSUPB574 $(\Delta q s m R)$ (Fig. 6D and E).

The expression of $q s m R$ was not affected by tofI/tofR

QS, while $q s m R$ partially affected the expression of tofR, toxJ, and toxR but not the expression of tofI.

To determine the impact of tofI/tofR QS on the expression of $q s m R$, the transcriptional level of $q s m R$ was accessed and compared between the wild type (336gr-1) and its $\Delta$ tofI-tofR
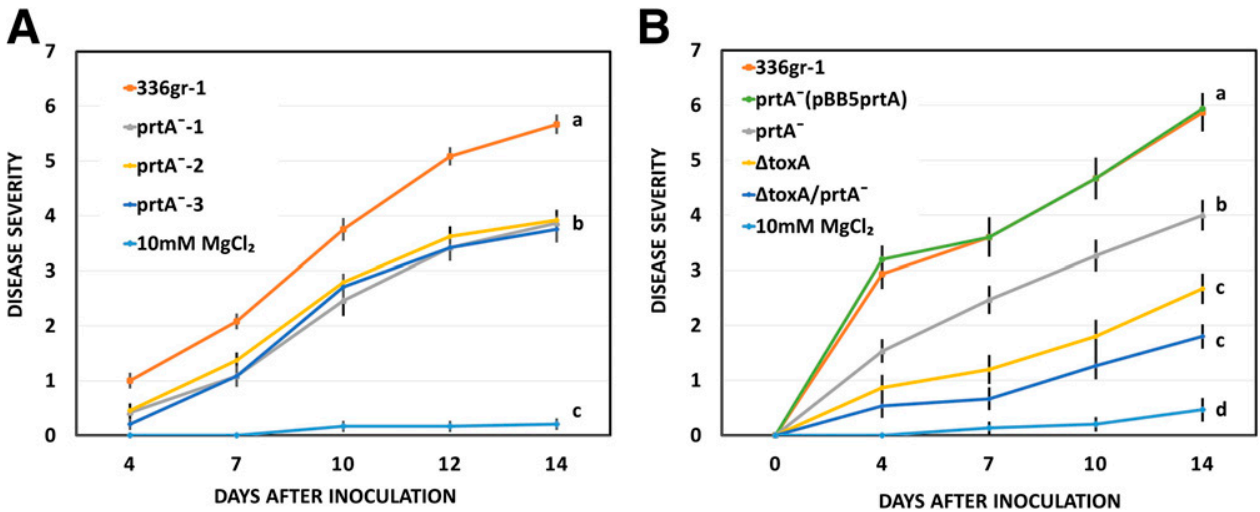

Fig. 4. The virulence function of prtA for Burkholderia glumae 336gr-1 in rice panicles. A, Disease progress curves of rice panicles inoculated with $B$. glumae 336gr- 1 and its three independent prtA $^{-}$derivatives, LSUPB586-1 (prtA $\left.A^{-}-1\right)$, LSUPB586-2 (prtA $A^{-} 2$ ) and LSUPB586-3 (prtA- 3 ). B, Disease progress curves of rice panicles inoculated with B. glumae 336gr-1 and its derivatives: LSUPB586-1 ( rrtA $^{-}$), LSUPB586-1 carrying a prtA clone [prtA ${ }^{-}$(pBB5prtA)], LSUPB537 ( $\triangle$ toxA), and LSUPB633 $\left(\triangle t o x A / p r t A^{-}\right)$. Disease severity was determined based on a 0 to 9 scale: $0=$ no symptom, $1=1$ to $10 \%$ symptomatic area, $2=11$ to $20 \%$ symptomatic area, $3=21$ to $30 \%$ symptomatic area, $4=31$ to $40 \%$ symptomatic area, $5=41$ to $50 \%$ symptomatic area, $6=51$ to $60 \%$ symptomatic area, $7=61$ to $70 \%$ symptomatic area, $8=71$ to $80 \%$ symptomatic area, and $9=$ more than $80 \%$ symptomatic area. The susceptible rice variety Trenasse was inoculated to evaluate the virulence of each strain. Error bars indicate the standard deviations from 15 replications. Similar results were also obtained from an additional two independent experiments. The letters at the end of individual data curves indicate statistically significant differences among the data curves at $P<0.05$, based on Tukey's posthoc test.
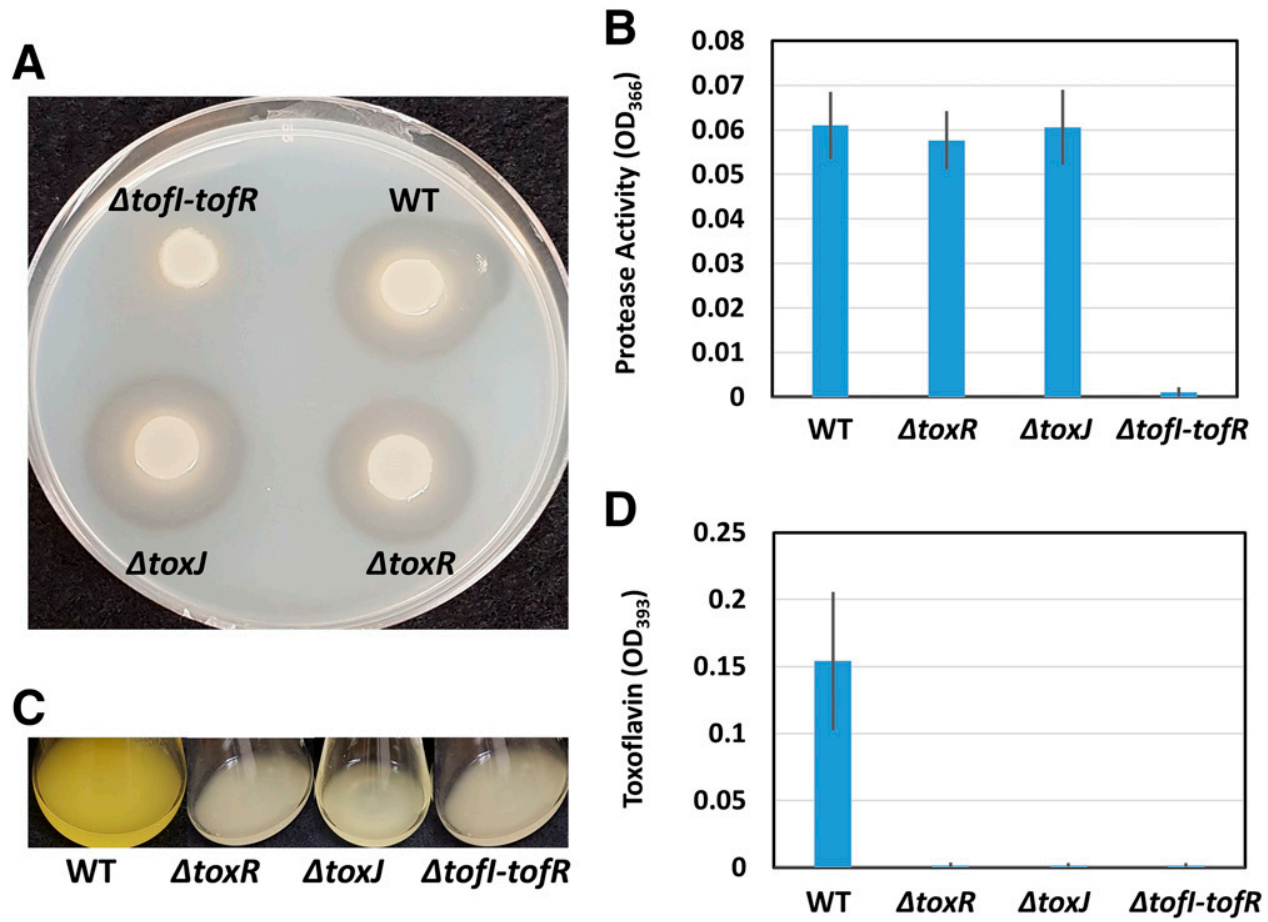

Fig. 5. The differential regulatory functions of toxJ and toxR in extracellular protease activity and toxoflavin production of Burkholderia glumae 336gr-1. A, Extracellular protease activities of the wild type (WT) and its $\Delta$ toxJ, $\Delta t o x R$, and $\Delta$ tofI-tofR derivatives on a nutrient agar plate supplemented with $1 \%$ skim milk. B, Quantified protease activities of the cell-free culture filtrates from the same set of B. glumae strains grown in Luria Bertani broth. Protein activity was quantified following the method of Chessa et al. (2000). C, Toxoflavin-producing phenotypes of the WT and its $\Delta$ toxJ, $\Delta$ toxR, and $\Delta t o f I-t o f R$ derivatives indicated by the yellow color of each cell culture. D, The toxoflavin-producing phenotypes of the same set of strains quantified based on observance at $393 \mathrm{~nm}$

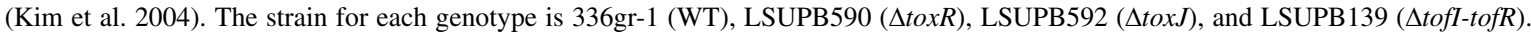


derivative (LSBPB139) with qRT-PCR. As shown in Figure 7A, there was no significant difference in the expression level of $q s m R$ between the wild type and its $\Delta t o f I-t o f R$ derivative, indicating that $q s m R$ expression is not affected by tofI/tofR QS in B. glumae 336gr-1. Likewise, tofI expression in the $\Delta q s m R$ genotype (LSUPB574) was not significantly different from that in the parent strain (336gr-1), indicating that $q s m R$ does not have a strong impact on the expression of tofI, either (Fig. 7B).
However, expression levels of other regulatory genes (tofR, toxJ, and toxR) were significantly reduced in the $\Delta q s m R$ derivative, indicating that $q s m R$, if not completely, partially regulates those regulatory genes (Fig. 7B). Meanwhile, expression of $\operatorname{toxA}$ (a structural gene for toxoflavin biosynthesis) was completely abolished in both $\Delta q s m R$ and $\Delta$ tofI-tofR backgrounds, which was congruent with the toxoflavin production phenotypes of these mutant strains (Fig. 7A and B).
A

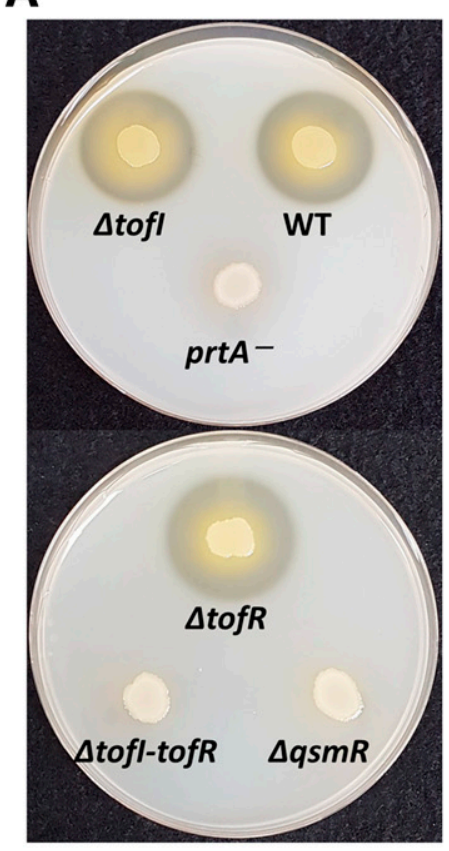

B

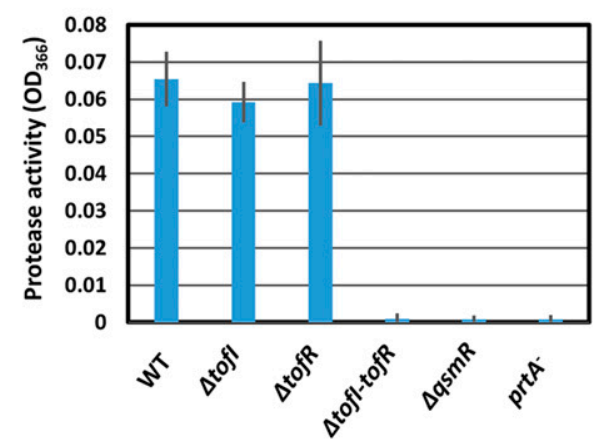

D

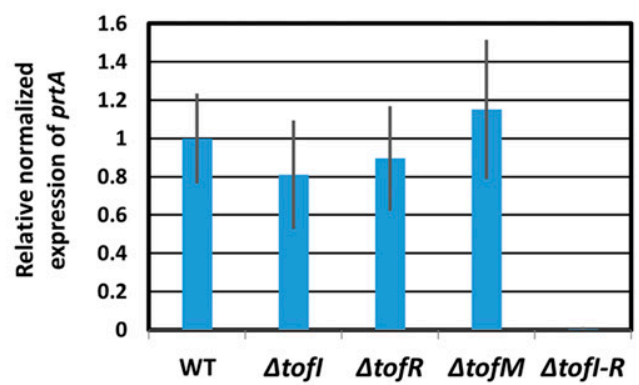

C

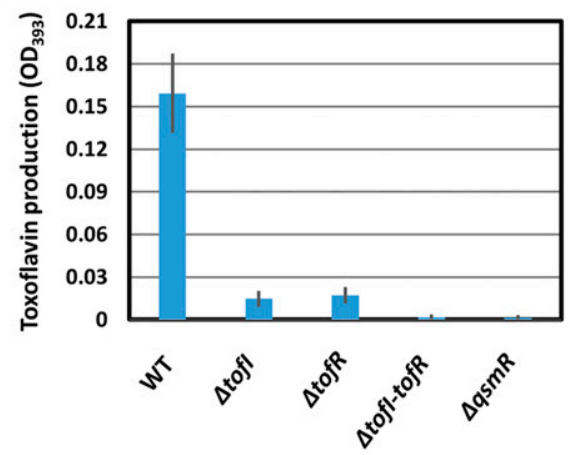

E

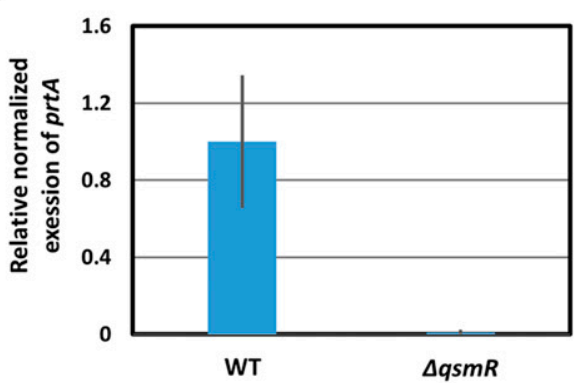

Fig. 6. The regulatory function of qsmR in extracellular protease activity and toxoflavin production of Burkholderia glumae 336gr-1. A, The extracellular protease activities of the wild type (WT) and its $\Delta q s m R, \Delta t o f I, \Delta t o f R, \Delta t o f I$-tofR, and $p r t A^{-}$derivatives on a nutrient agar plate supplemented with $1 \%$ skim milk. B, Quantified protease activities of cell-free culture filtrates from the same set of B. glumae strains grown in Luria Bertani broth. Protein activity was quantified following the method of Chessa et al. (2000). C, Toxoflavin-producing phenotypes of the WT and its $\Delta q s m R, \Delta t o f I, \Delta t o f R$, and $\Delta$ tofI-tofR derivatives, quantified based on observance at $393 \mathrm{~nm}$ (Kim et al. 2004). The strain for each genotype is $336 \mathrm{gr}-1$ (WT), LSUPB574 ( $\Delta q s m R$ ), LSUPB145 ( $\Delta$ tofI), LSUPB169 ( $\Delta$ tofR), LSUPB139 ( $\Delta$ tofI-tofR), and LSUPB586-1 ( $\left.p r t A^{-}\right)$. D, Expression levels of prtA determined by qRT-PCR in $336 g r-1$ (WT), LSUPB145

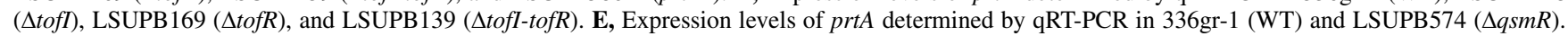

A

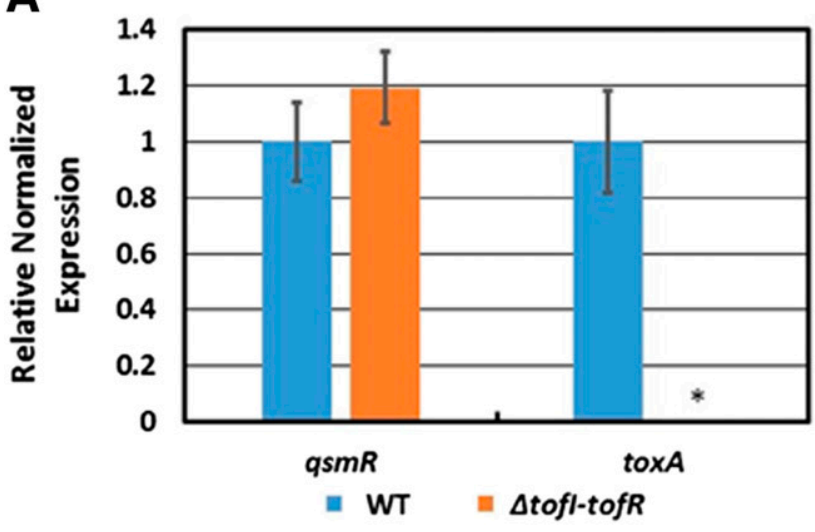

B

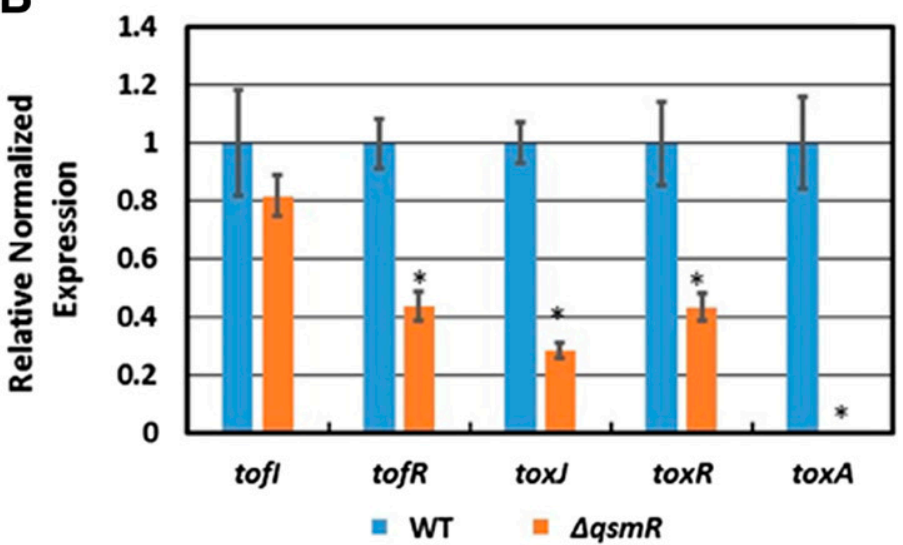

Fig. 7. Reciprocal influences between $q s m R$ and tofI/tofR determined by quantitative reverse transcription-polymerase chain reaction (qRT-PCR). A, The expression levels of $q s m R$ in the wild type (WT) and its $\Delta t o f I$-tofR derivative. B, The expression levels of tofI, tofR, toxJ, and toxR in the WT and its $\Delta q s m R$ derivative. Expression of toxA was also determined as a control for both A and B. The strain for each genotype is $336 \mathrm{gr}-1$ (WT), LSUPB139 ( $\Delta$ tofI-tofR), and LSUPB574 $(\Delta q s m R)$. Fold change of each gene in qRT-PCR was calculated by the $2^{-\Delta \Delta C t}$ method, and expression was normalized using the reference genes gyrA and $16 \mathrm{~S}$. Error bars represent standard deviation, and asterisks $(*)$ indicate significant differences among data at $P<0.05$, based on Tukey's studentized range test. 
Genetic elements involved in the extracellular protease activity of $B$. glumae 336gr-1 were identified through screening of random mini-Tn $5 \mathrm{Km}$ mutants.

Among approximately 4,000 random mutants screened on NA-1\% skim milk plates, six mutants showed significantly altered extracellular protease activity through repeated tests (Table 1). Among the six mutants screened, four mutants exhibited significantly reduced extracellular protease activities compared with the wild-type parent $336 \mathrm{gr}-1$, while two mutants did not show any detectable extracellular protease activity (Table 1). One of the four mutants showing reduced extracellular protease activities had a mini- $\mathrm{Tn} 5 \mathrm{Km}$ insertion in a putative gene likely involved in signal transduction (336gr-1mr4) (Table 1). Two extracellular protease-deficient strains, 336gr$1 \mathrm{mr} 1$ and $336 \mathrm{gr}-1 \mathrm{mr} 5$, were found to be disrupted in putative genes encoding a transcriptional regulator LysR family and an arginyl-tRNA-protein transferase, which are likely to be involved in transcriptional regulation process and posttranslational regulation, respectively (Table 1 ).

\section{DISCUSSION}

In this study, we found that extracellular protease activity, encoded by $\operatorname{prtA}$, is another critical virulence factor of $B$. glumae. According to the assay system used in this study, prtA appears to be solely responsible for the extracellular protease activity of $B$. glumae $336 \mathrm{gr}-1$, as determined by the diminished enzymatic activity of a prtA null mutant on NA-1\% skim milk plates. Analysis of the putative sequence of PrtA predicted the first $27 \mathrm{~N}$-terminus amino acids as an $\mathrm{N}$-terminal signal peptide for sec-dependent protein secretion, implying that this extracellular protease is secreted via a T2SS (Korotkov et al. 2012). Substantial reduction of the extracellular protease activity in the two T2SS-deficient mutant backgrounds ( $g s p D^{-}$ and $g s p E^{-}$) proved this notion, although residual enzymatic activities were detected with those secretion mutants (Fig. 2). As the T2SS exerts its function on the proteins exported from the cytoplasm to the periplasm via the sec or tat machinery, the observed residual activities are likely from the leakage of the PrtA proteases arrested in the periplasm of the $g s p D$ or $g s p E$ mutant. Blast search revealed the homologs of the PrtA protease among Burkholderia spp., including B. gladioli (another causal agent of BPB in rice) and other plant and animal pathogenic species (Supplementary Fig. S5). Interestingly, another serine metalloprotease (MrpA) is also present in B. glumae. However, this protease does not contain a signal sequence, suggesting that its enzymatic function is limited to the cytoplasm but not related to the extracellular protease activity of B. glumae.

A previous proteomic study by Goo et al. (2010) presented the idea that 34 extracellular proteins including the serine metalloprotease (prtA) were produced under the regulation of the tofI/tofR QS and were secreted via the T2SS in B. glumae BGR1. In that study, type II secretion-defective mutants $\left(g s p C^{-}, g s p E^{-}\right.$, and $\left.g s p J^{-}\right)$exhibited substantial reductions in disease severity, indicating the importance of the T2SSdependent extracellular proteins in the virulence of B. glumae BGR1. Our present study supports this and strongly suggests that prtA is a major virulence factor among the T2SS-dependent extracellular proteins of B. glumae.

Extracellular proteases play critical roles in bacterial pathogenesis (Frees et al. 2013; Lantz 1997), and several plant pathogens are known to rely on extracellular proteases for their pathogenic behaviors. A protease-deficient mutant of Xanthomonas campestris pv. campestris showed reduced ability to cause black rot systems on turnip leaves (Dow et al. 1990). In Xanthomonas oryzae pv. oryzicola, ecpA encoding an extracellular protease functions as a virulence factor in rice (Zou et al. 2012). Virulence functions of extracellular proteases were also reported in the studies of soft rot-causing plant-pathogenic bacteria (Marits et al. 1999; Shevchik et al. 1998). Interestingly, a recently characterized extracellular protease gene in Xylella fastidiosa, prtA, was found to act as an antivirulence factor, regulating cell growth, biofilm formation, and pathogenicity (Gouran et al. 2016), suggesting the complex functions of extracellular protease in bacterial pathogenesis. To the best of our knowledge, the present study demonstrates the virulence function of an extracellular protease in plant-pathogenic Burkholderia species for the first time, although the zinc metalloproteases $\mathrm{ZmpA}$ and $\mathrm{ZmpB}$ were previously reported as potential virulence factors of the animal pathogenic strains of B. cepacia and B. cenocepacia (Corbett et al. 2003; Kooi et al. 2006).

In B. glumae, major virulence factors are regulated by the QS system mediated by tofI and tofR (Chen et al. 2012; Chun et al. 2009; Kim et al. 2007). Results of this study indicated that the extracellular protease activity was also dependent upon the tofI/tofR QS. The deletion of the entire tofI/tofR QS gene cluster, including tofI, tof $M$, and $t o f R$, abolished the extracellular protease activity in the assay system used in this study. However, the single-gene mutants for the individual tofI, tofM, and tofR genes (LSUPB169, LSUPB 286, and LSUPB145, respectively) retained the enzymatic activity without any significant difference from wild type (Figs. 3 and 6). This observation is reminiscent of our previous data on toxoflavin production, in which toxoflavin production was completely inhibited by the deletion of the entire tofI/tofR QS gene cluster, while singlegene deletion mutants LSUPB145 ( $\Delta$ tofI) and LSUPB 169 $(\Delta t o f R$ ) still produced comparable amounts of toxoflavin on LB agar plates (Chen et al. 2012). In that study, deletion of tofM in the $\Delta t o f I$ or $\Delta t o f R$ background caused a substantial reduction of toxoflavin production on LB agar, suggesting that tof $M$ plays a role as a pivotal 'modulator' in the tofR- and tofI-independent production of toxoflavin production by LSUPB 145( $\Delta$ tofI) and LSUPB169 ( $\Delta t o f R)$, respectively. The results of extracellular protease activity and toxoflavin production in our present and previous studies suggest the presence of unknown signaling and regulatory pathways in $B$. glumae $336 \mathrm{gr}-1$ that regulate these virulence functions in conjunction with the tofI/tofR QS system including tofM (Fig. 8). The extracellular protease activity was not affected by toxJ and toxR, which are regulatory genes essential for toxoflavin production (Fig. 5), indicating that specific regulatory pathway for each virulence function branches out following the tofI/tofR QS. Collectively, it can be speculated that, in $B$. glumae 336gr-1, the two virulence functions (extracellular protease activity and toxoflavin production) share a common signaling and regulatory pathway involving the tofI and tofR genes, but decoupling also occurs conditionally in the

Table 1. Mini-Tn5Km mutants of Burkholderia glumae 336gr-1 showing altered phenotypes in extracellular protease activity

\begin{tabular}{|c|c|c|c|}
\hline Strain name & Locus tag ${ }^{a}$ & Phenotype $^{\text {b }}$ & Gene disrupted \\
\hline $336 \mathrm{gr}-1 \mathrm{rm} 1$ & bglu_1g34140 & NP & $\begin{array}{l}\text { Transcriptional regulator, } \\
\text { LysR family }\end{array}$ \\
\hline $336 \mathrm{gr}-1 \mathrm{rm} 2-1$ & bglu_1g31090 & & Excinuclease $\mathrm{ABC}$ subunit $\mathrm{A}$ \\
\hline $336 \mathrm{gr}-1 \mathrm{rm} 2-2$ & & - & \\
\hline $336 \mathrm{gr}-1 \mathrm{rm} 3$ & bglu_1g02410 & - & $\begin{array}{l}\text { Phenylacetate-CoA } \\
\text { oxygenase subunit PaaA }\end{array}$ \\
\hline $336 \mathrm{gr}-1 \mathrm{rm} 4$ & bglu_1g10190 & - & $\begin{array}{l}\text { Diguanylate } \\
\text { cyclase/phosphodiesterase }\end{array}$ \\
\hline 336gr-1rm5 & bglu_1g19530 & NP & $\begin{array}{l}\text { Arginyl-tRNA-protein } \\
\text { transferase }\end{array}$ \\
\hline
\end{tabular}

\footnotetext{
${ }^{a}$ Corresponding gene ID numbers of the reference genome BGR1.

b No extracellular protease activity (NP) and reduced extracellular protease activity (-) compared to wild-type parent 336gr-1.
} 
absence of tofI or tofR and in the downstream pathways specific to each virulence function. The regulatory pathway and its regulatory elements specific for the extracellular protease activity are currently being characterized, following the screening of random mutants with altered extracellular protease activity.

In previous seminal studies on B. glumae BGR1, qsmR was characterized to be dependent on the tofI/tofR QS for its transcription and to have a pleiotropic regulatory function in flagellar biogenesis for motility and virulence, as well as in metabolic modulation for survival in stress conditions (An et al. 2014; Goo et al. 2017; Jang et al. 2014; Kim et al. 2007). An RNA-seq study of B. glumae BGR1, performed by Kim et al. (2013), also supports those studies, in which tofI showed more a important role in motility than $q s m R$ did. In this study, however, transcription of $q s m R$ appeared to be independent of the tofII tofR QS in B. glumae 336gr-1 (Fig. 7A), indicating the presence of variation between the two B. glumae strains in the regulatory system. Another interesting fact found in this study was that $q s m R$ functions as an essential regulatory factor for toxoflavin production in B. glumae 336gr-1. In B. glumae BGR1, though disruption of $q s m R$ caused a substantial reduction of toxoflavin production in LB broth, the $q s m R$ mutant produced comparable amounts of toxofalvin to the wild-type parent in LB agar medium conditions (Kim et al. 2007). In B. glumae $336 \mathrm{gr}-1$, however, toxoflavin production was almost abolished in the $\Delta q s m R$ background under both LB broth and LB agar medium conditions, indicating that both $q s m R$ and the tofI/tofR QS are essential for this virulence function (Fig. 6C). Given the observation that $q s m R$ only partially regulates toxJ and toxR (Fig. 7B), qsmR may also exert its regulatory function through an unknown regulatory pathway required for toxoflavin production. $q s m R$ is also essential for the extracellular protease activity of $B$. glumae $336 \mathrm{gr}-1$, indicating its global regulatory role for multiple virulence functions like the tofI/tofR QS.

According to a schematic model based on results from this study, both tofI/tofR QS including tofM and $q s m R$ are required for toxoflavin production and extracellular protease activity
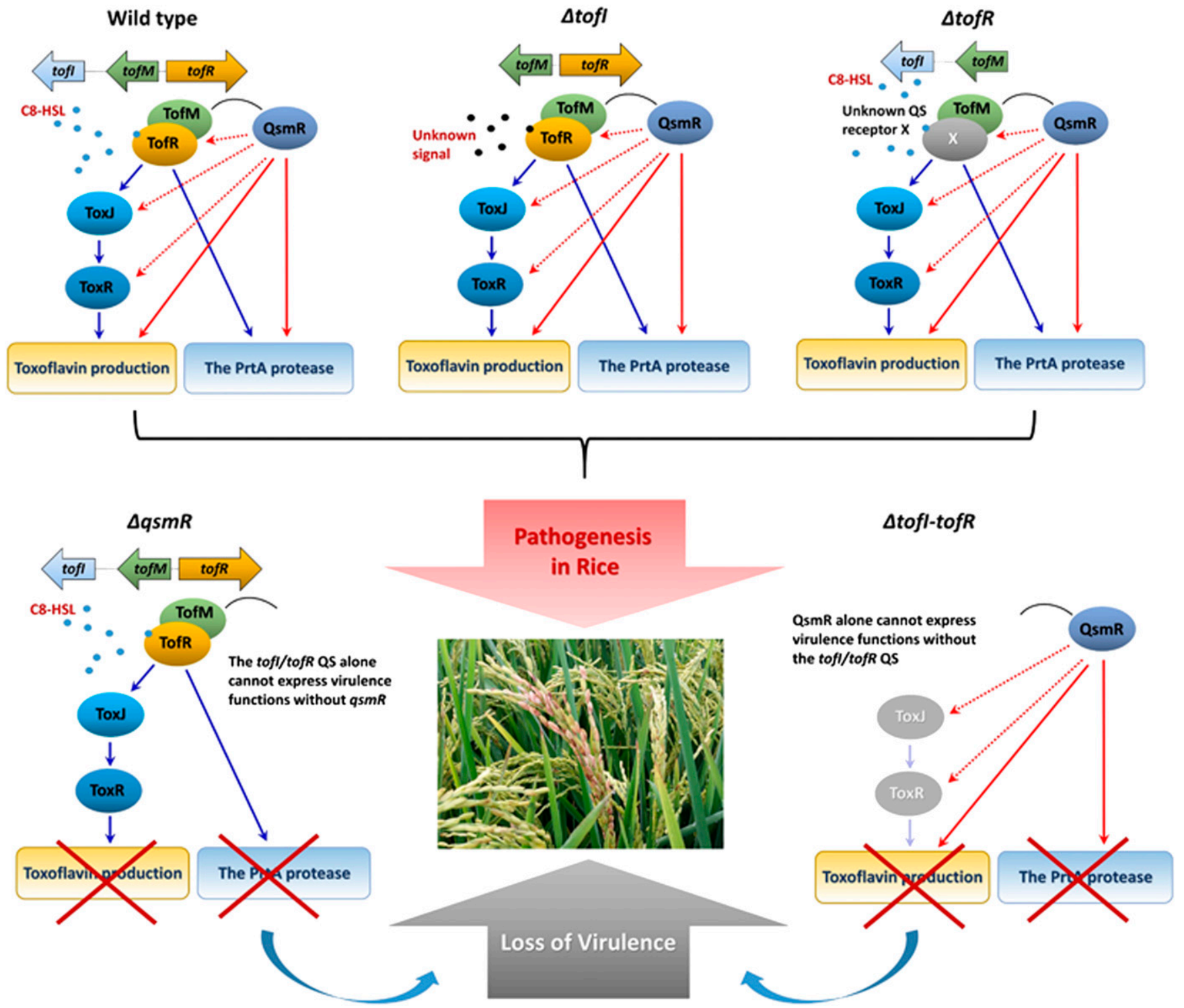

Fig. 8. A schematic model of the signaling/regulatory network for the virulence functions of Burkholderia glumae 336gr-1. The regulatory pathway through the tofI/tofR QS is indicated with blue arrows, while that through qsmR is indicated by red arrows. Partial influence of qsmR on the expression of tofR, toxJ, and toxR is indicated with hatched red arrows. According to the current model, both actions of the tofI/tofR QS and qsmR are required for the two virulence functions, toxoflavin production, and the PrtA protease activity. TofM is considered a pivotal modulator for the tofI/tofR QS function. C8-HSL $=$ octanoylhomoserine lactone (produced by tofI). 
(Fig. 8). In the absence of tofI (or C8-HSL), one or more unknown signals may replace the function of C8-HSL, leading to the production of toxoflavin and the PrtA extracellular protease (Fig. 8). Likewise, in the absence of tofR, one or more unknown receptors of C8-HSL may replace the function of TofR to express virulence functions (Fig. 8). However, in the tofI/tofR QSdeficient or $\Delta q s m R$ background, $q s m R$ or tofI/tofR QS alone, respectively, cannot make the expression of the virulence functions (Fig. 8). The unknown signaling molecules and the alternative QS signal receptor inferred from the model need to be identified through further comprehensive genetic and biochemical studies to elucidate the molecular mechanism underlying the expression of virulence functions in the absence of tofI or tofR.

The apparent difference between two strains of B. glumae (336gr1 versus BGR1) in the regulatory system involving the tofI/tofR QS and $q s m R$ implies that a certain degree of variation exists in the signaling and regulatory systems among different strains of $B$. glumae. We previously presented the diverse phenotypic variations related to virulence and pigmentation among strains of B. glumae (Karki et al. 2012). Considering that B. glumae 336gr-1 and B. glumae BGR1 belong in the same clade based on genomic structure and phenotypes (Karki et al. 2012), it is very probable that the diversity of the regulatory network for virulence functions within the entire $B$. glumae species is even more profound than that observed in this study between the two strains. In fact, we recently found that another virulent United States strain of B. glumae produces toxoflavin even in the absence of the tofI/tofR QS (T. Lelis and J. H. Ham unpublished).

In addition, candidate genetic elements involved in the extracellular protease activity of $B$. glumae were newly identified in this study through screening of random mini-Tn5Km mutants. All the mutants characterized showed reduced or nonprotease activities, suggesting that the mutated genes exert positive functions for the extracellular protease activity. At least some of the mutated genes identified in this study encode protein products known to function in signal transduction (e.g., diguanylate cyclase/phosphodiesterase), posttranslational regulation (e.g., arginyl-tRNA-protein transferase), and transcriptional regulation (e.g., LysR family transcriptional regulator), implying the high fidelity of this primary mutant screening process. Two other LysR family transcriptional regulators, toxR (Kim et al. 2004) and $n t p R$ (Melanson et al. 2017), were previously characterized in B. glumae. toxR regulates toxoflavin biosynthesis (Kim et al. 2004) (Fig. 5) but not the extracellular protease activity (Fig. 5). ntpR, on the other hand, is a negative regulator for toxoflavin production (Melanson et al. 2017), but its function on extracellular protease has not yet been determined. Nevertheless, further genetic studies are required to verify the regulatory function of each candidate gene identified in this study.

Conclusively, this study demonstrates i) the extracellular metalloprotease gene prtA is a new virulence factor of $B$. glumae, ii) the regulation pattern of extracellular protease activity is similar to that of the major virulence factor toxoflavin in the United States strain B. glumae 336gr-1, iii) qsmR functions as an essential regulatory gene for both extracellular protease activity and toxoflavin production in B. glumae 336gr1 , and iv) the global regulatory network involving the tofI/tofR QS and $q s m R$ varies significantly between $B$. glumae 336gr-1 and $B$. glumae BGR1. This study also strongly suggests that high levels of variation exist among different strains of $B$. glumae in the signaling and regulatory network for bacterial pathogenesis, which should be seriously considered when we attempt to design target-oriented management strategies for this pathogen based on its biological properties.

\section{MATERIALS AND METHODS}

\section{Strains and culture conditions.}

Bacterial strains and plasmids used in this study are listed in Supplementary Table S2. Bacterial strains were routinely grown in LB or LB agar medium, which was supplemented with antibiotics, as needed, at the following concentrations (per millimeter): $100 \mu \mathrm{g}$ of ampicillin, $50 \mu \mathrm{g}$ of kanamycin, $20 \mu \mathrm{g}$ of gentamycin, and $100 \mu \mathrm{g}$ of nitrofurantoin. DNA cloning and amplification were conducted following established methods (Sambrook and Russel 2001). PCR products were purified using the QuickClean 5M PCR purification kit (GenScript, Piscataway, NJ, U.S.A.). A NanoDrop ND-100 spectrophotometer (NanoDrop Technologies, Inc., Wilmington, DE, U.S.A.) was used to assess the quality and quantity of DNA samples. DNA sequencing of PCR products and DNA clones was performed by Macrogen USA (Rockville, MD, U.S.A.).

\section{Generation of $p r t A, g s p D$, and $g s p E$ mutants and the prtA clone for complementation.}

To generate a prtA::pKNOCK-Km mutant, a 403-bp internal fragment of $p r t A$ was amplified using MproTF1 and MprotR1 (Supplementary Table S3) and was ligated into the PCR cloning vector, psC-A-amp/kan. Using the EcoRI sites in the vector, the fragment was mobilized into the suicide vector pKNOCK-Km (Alexeyev 1999), generating pKnockprtA in Escherichia coli

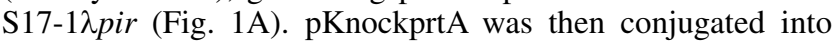
B. glumae via triparental mating, using the helper strain E. coli HB101 (pRK2013::Tn7). Colonies were selected based on antibiotic resistance to nitrofurantoin (for B. glumae) and kanamycin (for pKNOCK-Km inserted in prtA) on LB agar plates.

To complement the prtA::pKNOCK-Km mutant, 1,193-bp containing the promotor region and a $5^{\prime}$ portion of prtA and a 1,497-bp containing the remaining part of prtA were amplified using the MetprAupF1/MetprAupR1 and MetprAdownF1/MetprAdownR1 primer pairs, respectively (Fig. 1A). The upstream and downstream PCR products were ligated separately into the PCR cloning vector $\mathrm{pSC}-\mathrm{A}-\mathrm{amp} / \mathrm{kan}$, generating pTopometallUP and pTopometallDown, respectively. pTopometallDown was digested with NotI and $\mathrm{XbaI}$ and was subcloned into pKNOCK-Km suicide vector (Alexeyev 1999), generating pKkmMetallDown. pTopometallUP was digested with NotI and was cloned into pKkmMetallDown, generating pKkmprtA. pKkmprtA was digested with $E c o$ RI and was cloned into broad-host range vector pBBR1MCS-5 (Kovach et al. 1994), generating pBB5prtA (Fig.

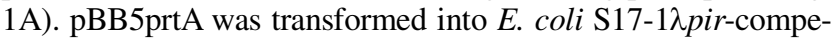
tent cells through electroporation and was then conjugated into B. glumae via triparental mating, using the E. coli HB101 (pRK2013::Tn7) helper strain. Colonies were selected based on resistance to nitrofurantoin and gentamycin on LB agar plates.

To generate a $g s p D:: p K N O C K-K m$ construct, a 541-bp internal fragment of $g s p D$ was amplified using TTssGspDF2 and TTssGspDR2. The resultant PCR product was then ligated to the PCR cloning vector psC-A-amp/kan. Using the EcoRI sites in the cloning vector, the cloned fragment was transferred to the suicide vector pKNOCK-Km (Alexeyev 1999), generating

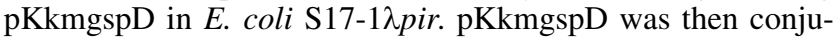
gated into B. glumae via triparental mating using the helper strain E. coli HB101 (pRK2013::Tn7). Colonies were selected based on the antibiotic resistance to nitrofurantoin (for B. glumae) and kanamycin (for pKNOCK-Km carrying the internal fragment of $g s p D$ ) on LB agar plates. To generate a gspE::pKNOCK-Km construct, a 468-bp internal fragment of gspE was amplified using TTssGspEF1 and TTssGspER1, followed by cloning into the PCR cloning vector psC-Aamp/kan. Using the EcoRI sites in the vector, the cloned 
fragment was transferred to the suicide vector pKNOCK-Km

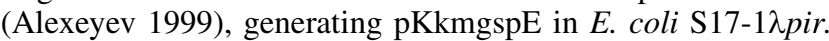
$g s p E$ was mutated in a same way as $g s p D$, using the DNA construct pKkmgspE.

\section{Generation of the $\Delta \operatorname{tox} A, \Delta \operatorname{tox} J$, and $\Delta$ tox $R$ derivatives of 336gr-1.}

To generate LSUPB537, a $\Delta$ toxA derivative of $336 \mathrm{gr}-1$, a 502-bp upstream flanking region, and a 481-bp downstream flanking region of toxA were amplified using primer sets ToxA UpF/ToxA UpR and ToxA DWNF/ToxA DWNR, respectively. The upstream and downstream PCR products were ligated separately into PCR cloning vector pSC-A-amp/kan. pSCtoxAup, the plasmid containing the upstream flanking sequence of toxA, was cleaved using BamHI and SpeI, and was then subcloned into the suicide vector pKKSacB (pKKSacBtoxAup). pSCtoxAdown, the downstream flaking sequence of toxA, was cleaved using SpeI and $X b a \mathrm{l}$ and was then cloned into pKKSacBtoxAup, generating pKKSacBtoxA. pKKSacBtoxA was transformed into $E$. coli $\mathrm{S} 17-1 \lambda$ pir competent cells through electroporation and was then conjugated into B. glumae via triparental mating, using E. coli HB101 (pRK2013::Tn7) helper strain. Bacterial cells having single homologous recombination were selected based on antibiotic resistance to nitrofurantoin and kanamycin on LB agar plates. Subsequently, the selected colonies were grown overnight at $30^{\circ} \mathrm{C}$ in $\mathrm{LB}$ broth without any antibiotics and were spread on LB agar plates containing 30\% sucrose to select mutants with secondary homologous recombination. Sucrose-resistant colonies were tested for sensitivity to kanamycin, and deletion of toxA was confirmed by PCR using the primer set ToxAcompF/ToxAcompR.

To generate LSUPB592, a $\Delta$ toxJ derivative of $336 \mathrm{gr}-1$, a 415-bp upstream flanking region and a 540-bp downstream flanking region of toxJ were amplified using primer sets ToxJUpFP/ToxJUpRP and ToxJDwnFP/ToxJDwnRP, respectively. Both upstream and downstream PCR fragments were ligated separately into the PCR cloning vector pSC-Aamp/kan. pTopotoxJup carrying the upstream sequence of toxJ was cleaved using the $X b a \mathrm{I}$ and $S p e I$ restriction sites, and the upstream sequence of toxJ was then subcloned into suicide vector $\mathrm{pKKSacB}$ to generate $\mathrm{pKKSacB}$ toxJup. pTopotoxJDWN carrying the downstream sequence of toxJ was cleaved using SpeI and BamHI, and the downstream sequence of toxJ was then ligated to pKKSacBtoxJup, generating pKKSacB $\Delta$ toxJ. pKKSacB $\Delta$ toxJ was transformed into

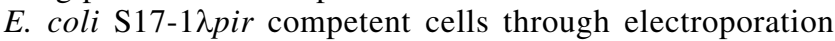
and was then conjugated into $B$. glumae via triparental mating, using the helper strain E. coli HB101(pRK2013:: $\mathrm{Tn} 7)$. Selection of $\Delta$ toxJ derivatives was achieved through the procedure previously established (Chen et al. 2012). Deletion of toxJ was confirmed by PCR using the primer set ToxJcompF/ToxJcompR.

The toxR gene sequence is present upstream of the tox operon containing five putative toxoflavin biosynthetic genes, toxA, toxB, tox $C$, toxD, and toxE. To generate LSUPB590, a $\Delta$ toxR derivative of $336 \mathrm{gr}-1$, the plasmid carrying a toxA upstream sequence, pKKSacBtoxAup, was used as the intermediate DNA construct for a toxR downstream sequence. For cloning an upstream region of toxR, a 408-bp upstream sequence of toxR was amplified using the primer set ToxRUpF/ToxRUpR. This $\mathrm{PCR}$ product was initially cloned in $\mathrm{pSC}-\mathrm{A}$-amp/kan, generating pTopotoxRup, and was subsequently ligated to pKKSacBtoxAup, using the BamHI restriction sites. The resultant DNA construct pKKSacB $\Delta$ toxR was used for generating $\Delta$ tox $R$ derivatives through the same procedure described above for deletion of toxJ. Deletion of toxR was confirmed by PCR using the primer set ToxRcompF/ToxRcompR.

\section{Generation of the $\Delta q s m R$ derivative of 336gr-1.}

A schematic illustration for $q s m R$ deletion is presented in Supplementary Figure S6. The markerless $q s m R$ deletion mutant LSUPB574 was generated through double-crossover homologous recombination in the flanking regions of the $q s m R$ gene. A 458-bp upstream region and a 469-bp downstream region were amplified with the primer sets qsmrBamHIUL/ qsmrSpeIUR and qsmrSpeIDL/qsmrXbalIDR1. Resultant PCR products of the upstream and downstream regions were then cloned to $\mathrm{pSC}$-A-amp/kan to generate $\mathrm{pSCqsmRU}$ and pSCqsmRD, respectively. The upstream region cloned in pSCqsmRU was further cloned to pKKSacB, using the BamHI and SpeI restriction sites to generate pKKqsmRU. Finally, the $q s m R$ downstream region present in pSCqsmRD was cloned to pKKqsmRU, using the SpeI and XbalI restriction sites to obtain $\mathrm{pKKSacB} \Delta \mathrm{qsmR}$. The DNA construct for deletion of $q s m R$, $\mathrm{pKKSacB} \Delta \mathrm{qsmR}$, was primarily transformed into E. coli $\mathrm{S} 17-$ $1 \lambda$ pir through electroporation and was then introduced into B. glumae, via triparental mating, using the helper strain E. coli HB101(pRK2013::Tn7). Selection of $\Delta$ toxJ derivatives was achieved through the procedure previously established (Chen et al. 2012). Deletion of $q s m R$ was confirmed by PCR using the primer set qsmrDLcheckF/qsmrDLcheckR.

\section{Extracellular protease assay.}

Extracellular protease activity was initially assessed on NA plates amended with $1 \%$ skim milk, following the method of Huber et al. (2001) with some modifications. Briefly, $1 \mathrm{ml}$ of an overnight culture of $B$. glumae incubated at $37^{\circ} \mathrm{C}$ was washed twice with fresh LB broth, using a microcentrifuge, and the final bacterial suspension was adjusted to an optical density at $600 \mathrm{~nm}\left(\mathrm{OD}_{600}\right)$ of 1.0. Five microliters of the bacterial suspension were spotted on an NA plate amended with $1 \%$ skim milk, followed by incubation at $37^{\circ} \mathrm{C}$ for $48 \mathrm{~h}$. Extracellular protease activity was determined based on the halo zone formed around each bacterial colony.

A quantitative assay of extracellular protease activity was conducted using azocasein (Sigma, Saint-Louis) as the proteolytic substrate, following the method of Chessa et al. (2000). Briefly, $100 \mu \mathrm{l}$ of the cell-free supernatant from an overnight bacterial culture grown in LB broth was combined with $400 \mu \mathrm{l}$ of the protease assay buffer (composed of $100 \mu \mathrm{l}$ of $30 \mathrm{mg}$ of azocasein per milliliter and $300 \mu \mathrm{l}$ of $20 \mathrm{mM}$ Tris/ $1 \mathrm{mM} \mathrm{CaCl}_{2}$, $\mathrm{pH} 8$ ). The reaction solutions were incubated for $10 \mathrm{~min}$ at $20^{\circ} \mathrm{C}$, followed by the addition of the stopping solution $(500 \mu \mathrm{l}$ of $100 \mathrm{mg}$ of trichloroacetic acid per milliliter). Following the subsequent centrifugation at $13,000 \times g$ for $2 \mathrm{~min}$, the absorbance of each supernatant was measured at $366 \mathrm{~nm}$, using a spectrophotometer (Biomate 3, Thermo Electron Corp.). Fresh medium without bacterial cells was used for the blank control.

\section{Toxoflavin quantification.}

Toxoflavin production was measured following the previously established method (Kim et al. 2004) with minor modifications. Briefly, bacterial strains freshly retrieved from glycerol stocks stored at $-80^{\circ} \mathrm{C}$ were grown in LB broth, for $24 \mathrm{~h}$ at $37^{\circ} \mathrm{C}$, in a shaking incubator rotating at $200 \mathrm{rpm}$. Each bacterial culture was then centrifuged to extract the toxoflavin present in the supernatant. For toxoflavin extraction, $1 \mathrm{ml}$ of the supernatant was mixed with $1 \mathrm{ml}$ of chloroform through vortexing for $15 \mathrm{~s}$. Each sample was centrifuged at $12,000 \times g$ for $10 \mathrm{~min}$ to separate the chloroform phase. The chloroform phase was transferred to a new microtube and was placed in a fume hood overnight to evaporate. After evaporation, the remaining materials in the microtube including toxoflavin were resuspended in $1 \mathrm{ml}$ of $80 \%$ methanol. Absorbance was measured at 
$393 \mathrm{~nm}\left(\mathrm{OD}_{393}\right)$, using the BioMate 3 spectrophotometer (Thermo Electron Corp.). Fresh medium without bacterial cells instead of a bacterial culture was used for the blank control.

\section{Virulence assay.}

Virulence assay was conducted with a highly susceptible rice variety, Trenasse. Overnight cultures of $B$. glumae strains on LB agar plates were suspended in sterile tap water to a final concentration of approximately $5 \times 10^{7} \mathrm{CFU} / \mathrm{ml}\left(\mathrm{OD}_{600}=0.1\right)$. Rice plants were inoculated twice in a 2-day interval with a hand sprayer when panicles were emerging. Disease progress was assessed five times during a 14-day period after inoculation. Statistical analysis for the virulence data sets was conducted with Tukey's post hoc tests using SAS version 9.3.

\section{RNA extraction, cDNA synthesis, and qPCR.}

B. glumae strains were cultured overnight in $10 \mathrm{ml}$ of LB broth at $37^{\circ} \mathrm{C}$ in a shaking incubator rotating at $200 \mathrm{rpm}$. A $1-\mathrm{ml}$ aliquot was washed twice with fresh LB broth and was resuspended in equal volumes of LB broth. Ten microliters of the resuspension were inoculated in $10 \mathrm{ml}$ of $\mathrm{LB}$ broth and were incubated at $37^{\circ} \mathrm{C}$ until bacterial culture reaches $\mathrm{OD}_{600}=1.0$. One milliliter of bacterial suspension was pelleted by centrifugation, was frozen in liquid nitrogen, and was resuspended in $1 \mathrm{ml}$ of TRIzol reagent (Ambion Life Technologies, Grand Island, NY, U.S.A.). RNA extraction and DNase treatment were performed using Direct-zolTM RNA MiniPrep kit (Zymo Research, Irvine, CA, U.S.A.), following manufacturer instructions. cDNA was prepared using iScript gDNA Clear cDNA synthesis kit (Bio-Rad Laboratories, Hercules, CA, U.S.A.), following manufacturer instructions. qPCR was performed using SsoAdvance Universal SYBR- Green supermix (Bio-Rad Laboratories, Inc.) following manufacturer instructions. Reactions were conducted in a Bio-Rad CFX Connect thermal cycler (Bio-Rad Laboratories, Inc.). The qPCR reaction program consisted of an initial denaturation step at $95^{\circ} \mathrm{C}$ for $60 \mathrm{~s}$, followed by 39 cycles of $95^{\circ} \mathrm{C}$ for $15 \mathrm{~s}$ and $52^{\circ} \mathrm{C}$ for $20 \mathrm{~s}$. A melt curve analysis of temperatures from $65^{\circ} \mathrm{C}$ to $95^{\circ} \mathrm{C}$ at $0.5^{\circ} \mathrm{C}$ increments was performed for each run. Expression values were normalized using two housekeeping genes, gyrA and $16 \mathrm{~S}$. Twotailed $t$ test was performed for the statistical analyses of the qRT-PCR data using SAS version 9.3.

\section{Sample preparation for RNA-seq.}

A 1-ml overnight culture of B. glumae 336gr-1 and LSUPB139 ( $\Delta$ tofI-R) (Chen et al. 2012) was washed twice and was resuspended in the same volume of LB broth, and $15 \mu \mathrm{l}$ of the suspension was inoculated in $15 \mathrm{ml}$ of $\mathrm{LB}$ and was incubated at $37^{\circ} \mathrm{C}$ until the early stationary phase $\left(\mathrm{OD}_{600}=1.0\right)$. Each bacterial culture $(1 \mathrm{ml})$ was then pelleted and placed in liquid nitrogen for 5 to $10 \mathrm{~s}$. The frozen pellet was resuspended in an equal volume of TRIzol reagent (Invitrogen) for RNA extraction. The extracted total RNA samples were then treated with DNA-free DNase treatment and removal reagents (Life Technologies) for the removal of residual DNA, following manufacturer instructions. RNA samples were then treated with a MEGAclear kit (Life Technologies) to eliminate the divalent cations left in the sample solution as well as the short oligonucleotides and protein molecules. RNA samples were then treated with a MICROBExpress bacterial mRNA enrichment kit (Life Technologies) for the removal of rRNA. The RNA samples were further processed with RNase III (Life Technologies) to reduce the fragmentation variability and with a RiboMinus Concentration Module (Life Technologies) to further eliminate rRNA. Library construction for RNA sequencing was performed using an RNA-Seq library construction kit (Life Technologies), following the manufacturer's instruction.
High-throughput DNA sequencing and sequence analysis.

High-throughput sequencing was conducted in the Virginia Bioinformatics Institute Core Lab, using the Illumina GAIIx platform for single-end sequencing for 50 cycles; $1.6 \mathrm{~GB}$ and 953.9 MB of sequence reads were generated from 336gr-1 and LSUPB139, respectively. To ensure high sequence quality, adaptors and low-quality sequences were trimmed using Trimmomatic (Bolger et al. 2014). The remaining sequences were mapped to the B. glumae BGR1 reference genome using Bowtie2 (Langmead and Salzberg 2012). Mapped reads were counted using HTSeq (Anders et al. 2015), and differential expression analyses were performed with edgeR (Robinson et al. 2010). Genes with a fold change in expression of $\geq 1.5$ and a false discovery rate of $\leq 0.05$ were considered to be differentially expressed.

\section{Random mutagenesis of $B$. glumae using mini-Tn5Km.}

Random mini-Tn5Km mutants of B. glumae were generated following a previously established method (Chen et al. 2012), through mixing a 3:1 ratio ( $\mathrm{vol} / \mathrm{vol})$ of E. coli S17-1 $\lambda$ pir (pUT:::mini-Tn5Km) (de Lorenzo et al. 1990) and B. glumae 336gr-1. Mutants were screened based on altered phenotypes compared with the parent in the halo zone formation on NA agar supplemented with $1 \%$ skim milk. The locations of mini-Tn $5 \mathrm{Km}$ insertion in the B. glumae genome were determined with a previously established method (Melanson et al. 2017), which was modified from the method described by Kwon and Ricke (2000).

\section{LITERATURE CITED}

Alexeyev, M. F. 1999. The pKNOCK series of broad-host-range mobilizable suicide vectors for gene knockout and targeted DNA insertion into the chromosome of gram-negative bacteria. Biotechniques 26:824-826, 828.

An, J. H., Goo, E., Kim, H., Seo, Y. S., and Hwang, I. 2014. Bacterial quorum sensing and metabolic slowing in a cooperative population. Proc. Natl. Acad. Sci. U.S.A. 111:14912-14917.

Anders, S., Pyl, P. T., and Huber, W. 2015. HTSeq-A Python framework to work with high-throughput sequencing data. Bioinformatics 31:166-169.

Bolger, A. M., Lohse, M., and Usadel, B. 2014. Trimmomatic: A flexible trimmer for Illumina sequence data. Bioinformatics 30:2114-2120.

Chen, R., Barphagha, I. K., Karki, H. S., and Ham, J. H. 2012. Dissection of quorum-sensing genes in Burkholderia glumae reveals non-canonical regulation and the new regulatory gene tof $M$ for toxoflavin production. PLoS One 7:e52150.

Chessa, J. P., Petrescu, I., Bentahir, M., Beeumen, J. Van Beeumen, and Gerday, C. 2000. Purification, physico-chemical characterization and sequence of a heat labile alkaline metalloprotease isolated from a psychrophilic Pseudomonas species. BBA-Protein Struct. M. 1479: 265-274.

Chun, H., Choi, O., Goo, E., Kim, N., Kim, H., Kang, Y., Kim, J., Moon, J. S., and Hwang, I. 2009. The quorum sensing-dependent gene kat $G$ of Burkholderia glumae is important for protection from visible light. J. Bacteriol. 191:4152-4157.

Corbett, C. R., Burtnick, M. N., Kooi, C., Woods, D. E., and Sokol, P. A 2003. An extracellular zinc metalloprotease gene of Burkholderia cepacia. Microbiology 149:2263-2271.

Cui, Z.-q., Zhu, B., Xie, G.-1., Li, B., and Huang, S.-w. 2016. Research status and prospect of Burkholderia glumae, the pathogen causing bacterial panicle blight. Rice Sci. 23:111-118.

de Lorenzo, V., Herrero, M., Jakubzik, U., and Timmis, K. N. 1990. MiniTn5 transposon derivatives for insertion mutagenesis, promoter probing, and chromosomal insertion of cloned DNA in gram-negative eubacteria. J. Bacteriol. 172:6568-6572.

Devescovi, G., Bigirimana, J., Degrassi, G., Cabrio, L., LiPuma, J. J., Kim, J., Hwang, I., and Venturi, V. 2007. Involvement of a quorum-sensingregulated lipase secreted by a clinical isolate of Burkholderia glumae in severe disease symptoms in rice. Appl. Environ. Microbiol. 73: 4950-4958

Dow, J. M., Clarke, B. R., Milligan, D. E., Tang, J. L., and Daniels, M. J. 1990. Extracellular proteases from Xanthomonas campestris pv. campestris, the black rot pathogen. Appl. Environ. Microbiol. 56:2994-2998.

Dunlap, P. V. 1999. Quorum regulation of luminescence in Vibrio fischeri. J. Mol. Microbiol. Biotechnol. 1:5-12. 
Francis, F., Kim, J., Ramaraj, T., Farmer, A., Rush, M. C., and Ham, J. H. 2013. Comparative genomic analysis of two Burkholderia glumae strains from different geographic origins reveals a high degree of plasticity in genome structure associated with genomic islands. Mol. Genet. Genomics 288:195-203.

Frees, D., Brøndsted, L., and Ingmer, H. 2013. Bacterial proteases and virulence. Subcell. Biochem. 66:161-192.

Goo, E., Kang, Y., Kim, H., and Hwang, I. 2010. Proteomic analysis of quorum sensing-dependent proteins in Burkholderia glumae. J. Proteome Res. 9:3184-3199.

Goo, E., Kang, Y., Lim, J. Y., Ham, H., and Hwang, I. 2017. Lethal consequences of overcoming metabolic restrictions imposed on a cooperative bacterial population. MBio 8:e00042-e17.

Goto, K., and Ohata, K. 1956. New bacterial diseases of rice (brown stripe and grain rot). Ann. Phytopathol. Soc. Jpn. 21:46-47.

Goto, M. 1992. Fundamentals of bacterial plant pathology. Academic Press, San Diego. CA, U.S.A.

Gouran, H., Gillespie, H., Nascimento, R., Chakraborty, S., Zaini, P. A., Jacobson, A., Phinney, B. S., Dolan, D., Durbin-Johnson, B. P., Antonova, E. S., Lindow, S. E., Mellema, M. S., Goulart, L. R., and Dandekar, A. M. 2016. The secreted protease PrtA controls cell growth, biofilm formation and pathogenicity in Xylella fastidiosa. Sci. Rep. 6: 31098.

Ham, J. H., Melanson, R. A., and Rush, M. C. 2011. Burkholderia glumae: Next major pathogen of rice? Mol. Plant Pathol. 12:329-339.

Huber, B., Riedel, K., Hentzer, M., Heydorn, A., Gotschlich, A., Givskov, M., Molin, S., and Eberl, L. 2001. The cep quorum-sensing system of Burkholderia cepacia H111 controls biofilm formation and swarming motility. Microbiology 147:2517-2528.

Jang, M. S., Goo, E., An, J. H., Kim, J., and Hwang, I. 2014. Quorum sensing controls flagellar morphogenesis in Burkholderia glumae. PLoS One 9:e84831.

Kang, Y., Kim, J., Kim, S., Kim, H., Lim, J. Y., Kim, M., Kwak, J., Moon, J. S., and Hwang, I. 2008. Proteomic analysis of the proteins regulated by HrpB from the plant pathogenic bacterium Burkholderia glumae. Proteomics 8:106-121.

Karki, H. S., Shrestha, B. K., Han, J. W., Groth, D. E., Barphagha, I. K., Rush, M. C., Melanson, R. A., Kim, B. S., and Ham, J. H. 2012. Diversities in virulence, antifungal activity, pigmentation and DNA fingerprint among strains of Burkholderia glumae. PLoS One 7: e45376.

Kim, J., Kang, Y., Choi, O., Jeong, Y., Jeong, J. E., Lim, J. Y., Kim, M., Moon, J. S., Suga, H., and Hwang, I. 2007. Regulation of polar flagellum genes is mediated by quorum sensing and FlhDC in Burkholderia glumae. Mol. Microbiol. 64:165-179.

Kim, J., Kim, J. G., Kang, Y., Jang, J. Y., Jog, G. J., Lim, J. Y., Kim, S., Suga, H., Nagamatsu, T., and Hwang, I. 2004. Quorum sensing and the LysR-type transcriptional activator ToxR regulate toxoflavin biosynthesis and transport in Burkholderia glumae. Mol. Microbiol. 54: 921-934.

Kim, S., Park, J., Kim, J. H., Lee, J., Bang, B., Hwang, I., and Seo, Y. S. 2013. RNAseq-based transcriptome analysis of Burkholderia glumae quorum sensing. Plant Pathol. J. 29:249-259.

Kooi, C., Subsin, B., Chen, R., Pohorelic, B., and Sokol, P. A. 2006. Burkholderia cenocepacia $\mathrm{ZmpB}$ is a broad-specificity zinc metalloprotease involved in virulence. Infect. Immun. 74:4083-4093.

Korotkov, K. V., Sandkvist, M., and Hol, W. G. 2012. The type II secretion system: Biogenesis, molecular architecture and mechanism. Nat. Rev. Microbiol. 10:336-351.
Kovach, M. E., Phillips, R. W., Elzer, P. H., Roop, R. M., 2nd, and Peterson, K. M. 1994. pBBR1MCS: A broad-host-range cloning vector. Biotechniques 16:800-802.

Kwon, Y. M., and Ricke, S. C. 2000. Efficient amplification of multiple transposon-flanking sequences. J. Microbiol. Methods 41:195-199.

Langmead, B., and Salzberg, S. L. 2012. Fast gapped-read alignment with Bowtie 2. Nat. Methods 9:357-359.

Lantz, M. S. 1997. Are bacterial proteases important virulence factors? J. Periodontal Res. 32:126-132.

Lim, J., Lee, T. H., Nahm, B. H., Choi, Y. D., Kim, M., and Hwang, I. 2009. Complete genome sequence of Burkholderia glumae BGR1. J. Bacteriol. 191:3758-3759.

Marits, R., Kõiv, V., Laasik, E., and Mäe, A. 1999. Isolation of an extracellular protease gene of Erwinia carotovora subsp. carotovora strain SCC3193 by transposon mutagenesis and the role of protease in phytopathogenicity. Microbiology 145:1959-1966.

Melanson, R. A., Barphagha, I., Osti, S., Lelis, T. P., Karki, H. S., Chen, R., Shrestha, B. K., and Ham, J. H. 2017. Identification of new regulatory genes involved in the pathogenic functions of the rice-pathogenic bacterium Burkholderia glumae. Microbiology 163:266-279.

Nandakumar, R., Shahjahan, A. K. M., Yuan, X. L., Dickstein, E. R., Groth, D. E., Clark, C. A., Cartwright, R. D., and Rush, M. C. 2009. Burkholderia glumae and B. gladioli cause bacterial panicle blight in rice in the southern United States. Plant Dis. 93:896-905.

Nickzad, A., Lépine, F., and Déziel, E. 2015. Quorum sensing controls swarming motility of Burkholderia glumae through regulation of rhamnolipids. PLoS One 10:e0128509.

Overbeek, R., Begley, T., Butler, R. M., Choudhuri, J. V., Chuang, H. Y., Cohoon, M., de Crécy-Lagard, V., Diaz, N., Disz, T., Edwards, R., Fonstein, M., Frank, E. D., Gerdes, S., Glass, E. M., Goesmann, A., Hanson, A., Iwata-Reuyl, D., Jensen, R., Jamshidi, N., Krause, L., Kubal, M., Larsen, N., Linke, B., McHardy, A. C., Meyer, F., Neuweger, H., Olsen, G., Olson, R., Osterman, A., Portnoy, V., Pusch, G. D., Rodionov, D. A., Rückert, C., Steiner, J., Stevens, R., Thiele, I., Vassieva, O., Ye, Y., Zagnitko, O., and Vonstein, V. 2005. The subsystems approach to genome annotation and its use in the project to annotate 1000 genomes. Nucleic Acids Res. 33:5691-5702.

Petersen, T. N., Brunak, S., von Heijne, G., and Nielsen, H. 2011. SignalP 4.0: Discriminating signal peptides from transmembrane regions. Nat. Methods 8:785-786.

Robinson, M. D., McCarthy, D. J., and Smyth, G. K. 2010. edgeR: A Bioconductor package for differential expression analysis of digital gene expression data. Bioinformatics 26:139-140.

Sambrook, J. and Russel, D. W. 2001. Molecular Cloning: A Laboratory Manual, 3rd ed. Cold Spring Harbor Press, Cold Spring Harbor, NY, U.S.A. Sandkvist, M. 2001. Type II secretion and pathogenesis. Infect. Immun. 69: 3523-3535.

Shevchik, V. E., Boccara, M., Vedel, R., and Hugouvieux-Cotte-Pattat, N. 1998. Processing of the pectate lyase PelI by extracellular proteases of Erwinia chrysanthemi 3937. Mol. Microbiol. 29:1459-1469.

Tsushima, S. 1996. Epidemiology of bacterial grain rot of rice caused by Pseudomonas glumae. Jpn. Agric. Res. Q. 30:85-89.

Zou, H. S., Song, X., Zou, L. F., Yuan, L., Li, Y. R., Guo, W., Che, Y. Z., Zhao, W. X., Duan, Y. P., and Chen, G. Y. 2012. EcpA, an extracellular protease, is a specific virulence factor required by Xanthomonas oryzae pv. oryzicola but not by X. oryzae pv. oryzae in rice. Microbiology 158 : 2372-2383. 